\title{
Big Data-Survey
}

\author{
PSG Aruna Sri ${ }^{* 1}$, Anusha $\mathbf{M}^{2}$ \\ ${ }^{1}$ Department of Electronics and Computer Engineering, $\mathrm{K} L$ University, \\ ${ }^{2}$ Research Scholar, Dept of CSE, K L UNIVERSITY \\ Greenfields, Vaddeswaram, Guntur District, Andhra Pradesh 522502 \\ ${ }^{*}$ Corresponding author, e-mail: arunasri_2012@kluniversity.in ${ }^{1}$, anushaaa9@kluniversity.in²
}

\begin{abstract}
Big data is the term for any gathering of information sets, so expensive and complex, that it gets to be hard to process for utilizing customary information handling applications. The difficulties incorporate investigation, catch, duration, inquiry, sharing, stockpiling, Exchange, perception, and protection infringement. To reduce spot business patterns, anticipate diseases, conflict etc., we require bigger data sets when compared with the smaller data sets. Enormous information is hard to work with utilizing most social database administration frameworks and desktop measurements and perception bundles, needing rather enormously parallel programming running on tens, hundreds, or even a large number of servers. In this paper there was an observation on Hadoop architecture, different tools used for big data and its security issues.
\end{abstract}

Keywords: Big data, Hadoop, Software tools, Map Reduce

\section{Introduction}

We make 2.5 quintillion bytes of information [1] - so much that $90 \%$ of the data on the planet today has been made in the last two years alone. This data originates from all around: sensors used to accumulate atmosphere data, posts to social media sites, digital pictures and videos, and cell phone GPS signal. This enormous amount of the data is known as "Big data". Big data is a catchphrase, or motto, utilizes to describe a massive volume of both structured and unstructured data that is so huge that it's complicated to process using conventional database and software procedures. In most project circumstances the information is too big or it shifts too quickly or it surpasses existing processing ability.

Big data has the potential to help organizations to improve operations and make faster by taking more intelligent decisions. Now-a-days, Big Data is the term which finds to be normal in IT businesses. As there was enormous information in the industry although there is nothing before big data which comes into imagine. Big data is really an advancing term that illustrates any huge amount of organized, semi organized information that can possibly be extracting for data. Although big data doesn't refer to any particular quantity, so this term is often utilized when talking about petabytes and exabytes of data Big data is a comprehensive term for expansive accumulation of the data sets so this large and complex that it gets to be troublesome to work with conventional data processing applications. At the point when managing bigger datasets, organizations face challenges in creating and managing big data. No standard tools and procedures for searching and analyzing large data sets in business analytics A case of Big data may be petabytes (1,024 terabytes) or exabytes $(1,024$ petabytes) of data comprising of billions to trillions of records of a huge number of individuals all from distinctive sources for example agreements, web, moveable data. The data is commonly approximately organized data that is frequently fragmented also, inaccessible. The problems faced by big data are analyzing, capturing, searching, sharing, storing, transferring, visualization and privacy abuse. Larger data sets is needed in order to prevent diseases, combat crime, spot business trends and so on. Because of large information sets in these area researchers identify limitations frequently like meteorology, genomics, connectomics, complex physics simulations, biological, ecological research, and funding and trade information. Data sets increased their size due to collecting data from sensing portable devices, aerial sensory technology, software logs, cameras, microphones, radio-frequency identification readers, and wireless sensor networks.

In March 2012 [2], the Obama administration announced the big data research and development initiative. The leading IT companies, such as SAG, Oracle, IBM, Microsoft, SAP 
and HP, have spent more than $\$ 15$ billion on buying data management and analytics software. Big data defined as far back as 2001, industry expert Doug Laney (right now with Gartner) expressed the standard meaning of big data as the $5 \mathrm{Vs}$ of big data: volume, velocity, variety, veracity and value, as shown Figure 1.

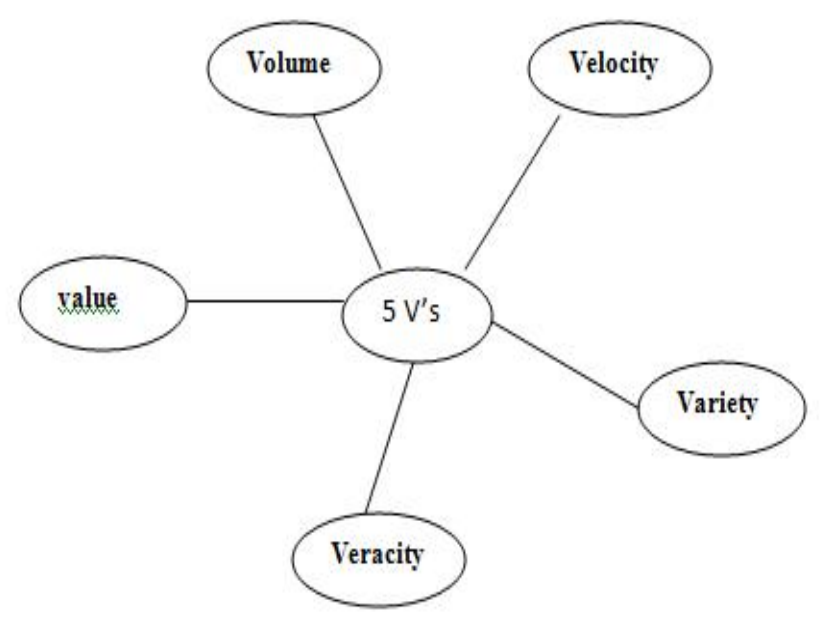

Figure 1. Five (5) V's factor of big data

Volume: At present big scale of systems are flooded with constantly increasing information, simply growing terabytes or even petabytes of data.

Velocity: Information is flowing at unique speed and should be deal with a sensible way. In real time for many organizations it is difficult to deal with RFID tag, sensors and smart metering data. Variety: Organized and unorganized information are producing a variety of data types by making it feasible to search novel approaches, while analyzing these information collectively, prediction might be attained as data flow into the organization.

Veracity: Identifying and verifying inconsistent information is significant, to accomplish faithful study. Creating faith in big data is a big challenge to manage even more variety of data is available.

Value: It is to be derived from big data. There is no reason for building the capacity to store and manage, if unable to get the value from data.

One of the capable remarks on the advances of the arrangement with the Big Data is Hadoop.

\section{Hadoop}

Hadoop was made by Doug Cutting and Mike Cafarella in 2005. Doug Cutting, who was working at Yahoo! at the time, named it after his kid's toy elephant.

Hadoop system is shown Figure 2 and consists [3]:

Reliable: this software can handle both hardware and software failures.

Scalable: Designed for gigantic size of processors, memory, and local appended capacity Distributed: Map reduce recommends parallel programming model and provides concept of replication 


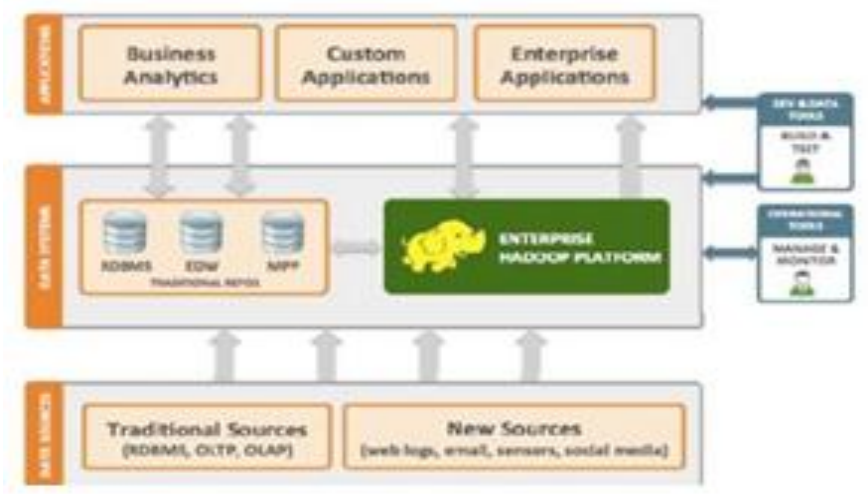

Figure 2. Hadoop system [1]

Hadoop is open-source programming that allows loyal, flexible, expressed figuring on groups of reserved servers. That utilization the Map-Reduce framework presented by Google by utilizing the idea of map and reduce functions that remarkable utilized in Functional Programming. In spite of the fact that the Hadoop structure is composed in Java, it permits designers to send custom-composed projects coded in Java or some other dialect to process information in a parallel manner over hundreds or a great many thing servers. It is streamlined for touching read requests (streaming reads), where transforming incorporates of checking all the information. Contingent upon the intricacy of the procedure and the volume of information, reaction time can change from minutes to hours. While Hadoop can forms information quick, so its key focal point is its monstrous adaptability. Hadoop is right now being utilized for file web seeks, email spam location, proposal motors, expectation in budgetary administrations, genome control in life sciences, furthermore, for investigation of unstructured information, for example, log, content, and click stream. While a considerable lot of these applications could indeed be actualized in a social database (RDBMS) figure 2, the primary center of the Hadoop system is practically not the same as a RDBMS. The accompanying examines some of these distinctions Hadoop is especially helpful when:

- For handling of Complex data.

- Need of conversion of unstructured information to structured information

- Usage of SQL queries

- Recursive calculations are very large

- Complex geo-spatial investigation or genome sequencing

- Machine learning

- Data sets are so substantial it couldn't be possible fit into database RAM, plates, or require an excess of centers (10's of TB up to PB)

- Data worth does not defend cost of steady ongoing accessibility, for example, files or exceptional interest information, which can be moved to Hadoop and stay accessible at lower expense

- Results are not required continuously

- Fault resistance is basic

- Significant custom coding would be obliged to handle employment booking

Hadoop was motivated by Google's Map Reduce, a product structure in which an application is separated into various little parts. Any of these parts (additionally called sections or squares) can be run on any hub in the group. Doug Cutting, Hadoop's maker, named the system after his youngster's full toy elephant. The current Apache Hadoop biological system comprises of the Hadoop bit, Map Reduce, the Hadoop circulated record framework (HDFS) what's more, various related activities, for example, Apache Hive, HBase and Zookeeper. The Hadoop structure is utilized by significant players including Google, Yahoo and IBM, generally for applications including web search tools and promoting. The favored working frameworks are Windows and Linux be that as it may Hadoop can likewise work with BSD and OS X. 
A distributed file system framework is a customer/server-based application that permits customers to get to and process information put away on the server as though it were all alone PC. At the point when a client gets to a document on the server, the server sends the client a duplicate of the document, which is stored on the client's PC while the information is being prepared and is then come back to the server. Preferably, a convey record framework arranges document and registry administrations of individual servers into a worldwide catalog in such a way that remote information access is not area particular however is indistinguishable from any customer. All records are available to all clients of the worldwide record framework and association is progressive what more, registry based is. Since more than one customer may get to the same information all the while, the server must have a system in spot, (for example, keeping up data about the times of access) to arrange overhauls so that the customer dependably gets the most current adaptation of information and that information clashes don't emerge. Dispersed record frameworks ordinarily utilize record or database replication (conveying duplicates of information on different servers) to ensure against information access failures [4]. Sun Microsystems' Network File System (NFS), Novell NetWare, Microsoft's Distributed File Framework, and IBM/Transarc's DFS are a few samples of distributed file systems framework.

\section{HDFS}

Hadoop frame work consists the Hadoop Distributed File System (HDFS) [4]. HDFS is planned and improved to store information more than a lot of ease equipment in an appropriated manner, as shown in Figure 3.

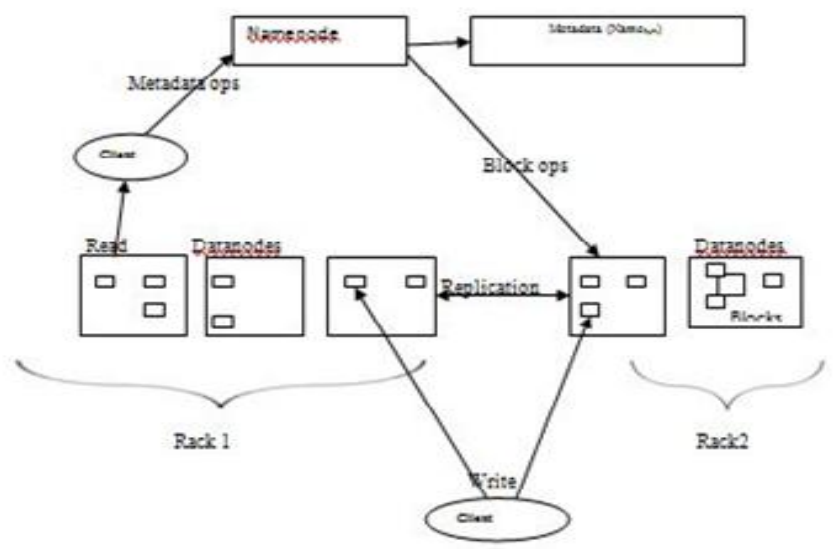

Figure 3. HDFS architecture

The structure of HDFS is master/slave. HDFS cluster consists of one Name Node, a master server that manages the classification system namespace and regulates access to files by clients. In addition, there square measure variety of information nodes, sometimes one per node within the cluster, that manage storage hooked up to the nodes that they run on. HDFS exposes a classification system namespace and permits user knowledge to be hold on in files. Internally, a file is split into one or a lot of blocks and these blocks square measure hold on during a set of information Nodes. The Name Node executes classification system namespace operations like opening, closing, and renaming files and directories. It conjointly determines the mapping of blocks to knowledge Nodes. The info Nodes square measure to responsibility for serving scans and write requests from the file system's clients. The info Nodes conjointly performs block formation, deletion, and replication upon instruction from the Name Node.

The Name Node and knowledge Node square measure items of package designed to run on goods machines. These machines generally run a GNU/Linux software system (OS). HDFS is constructed exploitation the Java language; any machine that supports Java will run the Name Node or the Data Node package. Usage of the extremely moveable Java language 
implies that HDFS will be deployed on a large variety of machines. A typical readying includes a dedicated machine that runs solely the Name Node package. Every of the opposite machines within the cluster runs one instance of the Data Node package. The design doesn't preclude running multiple knowledge Nodes on an equivalent machine however during a real readying that's seldom the case. The existence of Name Node during a cluster greatly simplifies the design of the system. The Name Node is that the intermediary and repository for all HDFS data. The system is intended to flow the user knowledge through the Name Node.

The base Apache Hadoop structure is made out of the taking after modules: Hadoop Common-contains libraries and utilities required by other Hadoop modules. Hadoop Distributed File System (HDFS) - a appropriated document framework that stores information on product machines, giving high total data transfer capacity over the group. Hadoop Map Reduce - a programming model for huge scale information preparing. All the modules in Hadoop are planned with a basic presumption that equipment disappointments (of individual machines, or racks of machines) are normal also, consequently ought to be naturally taken care of in programming by the structure. Apache Hadoop's Map Reduce and HDFS parts initially got individually from Google's Map Reduce and Google File System (GFS) papers."Hadoop" frequently alludes not to simply the base Hadoop bundle yet rather to the Hadoop Ecosystem fig.4 which incorporates the greater part of the extra programming bundles that can be introduced on top of or nearby Hadoop, for example, Apache Hive, Apache Pig and A HBase.

\section{Map Reduce Framework}

Map Reduce as shown in Figure 4 is a product system for appropriated transforming of Big data sets on PC groups [5]. It is first grown by Google. Map Reduce is planned to encourage also, improve the preparing of incomprehensible measures of information in parallel on extensive bunches of merchandise equipment in a solid, issue tolerant way. Map Reduce is the key calculation that the Hadoop Map Reduce motor uses to circulate work around a bunch. Commonplace Hadoop bunch coordinates Map Reduce and HFDS layer. In Map Reduce layer job tracker assigns tasks to the task tracker. Master node job tracker also allots tasks to the slave node task tracker figure.

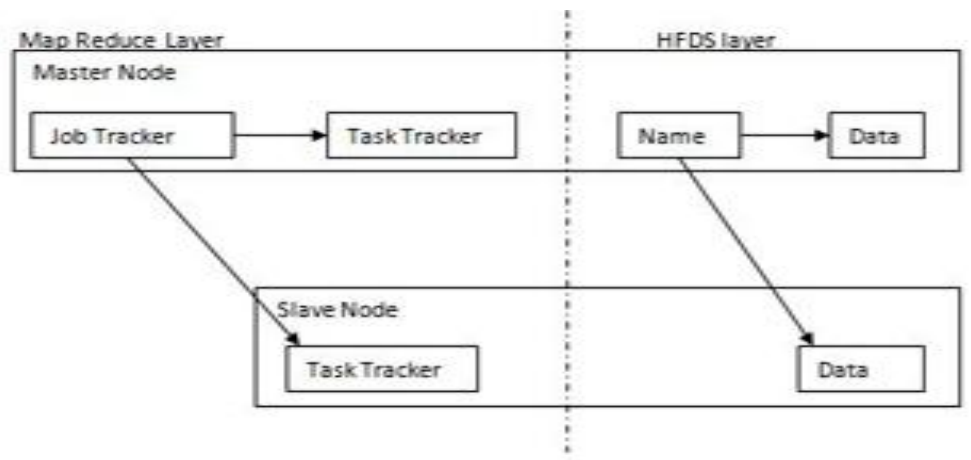

Figure 4. Map reduce is based on the Master-Slave architecture

Master node contains -

- Job tracker node (Map Reduce layer)

- Task tracker node (Map Reduce layer)

- Name node (HFDS layer)

- Data node (HFDS layer)

Multiple slave nodes contain -

- Task tracker node (Map Reduce layer)

- Data node (HFDS layer)

- Map Reduce layer has job and task tracker nodes

- HFDS layer has name and data nodes

A. Map Reduce core functionality (I):

Map \& Reduce stage plays an significant role in map reduce core functionality. 
Map stage: In Map step, master node takes consideration of large problem input and divided into smaller problems and allotted to worker nodes. These nodes process the smaller problems and return to the master node.

- Map (key1, value) $\Rightarrow$ list<key2, value2>

Reduce stage: In this Reduce stage, Master node takes the response from the sub problems and joins them in a predefined manner to get the output to original problem, as shown in Figure 5.

- Reduce (key2, list $<$ value2 $>$ ) $\Rightarrow$ list

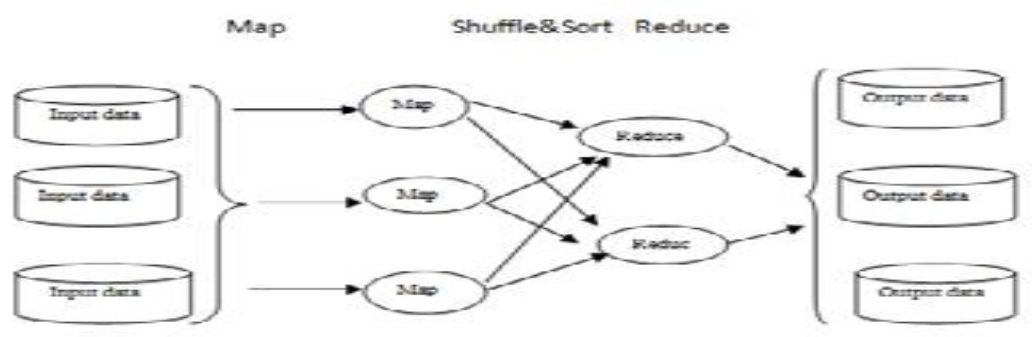

Figure 5. Reduce stage

\section{PIG}

Pigs [6] was at first shaped at Yahoo! to permit individuals utilizing Hadoop to concentrate all the more on examining substantial information sets and invest less moment of time is needed to compose mapper and reducer programs. Pig is comprised of two segments: the first is the is called Pig Latin and the second is a runtime situation where Pig Latin programs are executed.

\section{HIVE}

Apache Hive [7] was initially developed by face book. It has data warehouse structure built on top of hadoop for analysis and inquiry of data. By default, Hive stores metadata in an installed Apache Derby Database and other customer/server databases like MySQL can alternatively be used. Right now, there are four document configurations upheld in Hive, which are TEXTFILE SEQUENCEFILE, ORC and RCFILE.

\section{HBase}

HBase [8] is a section arranged database administration framework that keeps running on top of HDFS. HBase applications are composed in Java much like an average Map Reduce application. A HBase framework consists an arrangement of tables. Table Consists of rows and columns like a conventional database.

\section{Issues}

Although a considerable measure of research is going on big data yet at the same time. Several ideas are still to be investigated. Scientists would attempt to upgrade security stage to enhance capacity of programming to discover propelled dangers, respond in like manner and would create preventive measures for future. Specialists would attempt to enhance quality and dependability of security framework. A few analysts are wanting to taken up information accumulation, pretreatment, incorporation, Map Reduce and investigation utilizing machine learning strategies. They would utilize the outcomes for securing and actualizing preventive measures from dangers to big business information. Specialists would attempt to outline the meet the creation needs of endeavors for growing high caliber item by applying efforts to 
establish safety with the assistance of big data Analytics with Hadoop. A few specialists are utilizing systems administration checking tools like Packet pig, Mahout and so on to Improve the security levels. Targeted threats will be analyzed by using hadoop cluster.

\section{Conclusion}

Big data is going to keep developing amid the following years, and every information researcher will need to oversee considerably more measure of information will be more different, bigger, and speedier. We talked about a few experiences about the theme, and what we consider are the fundamental concerns and primary issues for what's to come. Big data is turning into new final boundary for experimental information research and for business applications. Everyone is warmly welcomed to take part in this fearless trip.

\section{References}

[1] Ms Vibhavari Chavan et al. "Survey Paper on Big Data". International Journal of Computer Science and Information Technologies. 2014; 5 (6), ISSN: 0975-9646.

[2] Michael R Lyu. "Service-generated Big Data and Big Data-as-a-Service". Internetware. 2013.

[3] Suman Arora et al. "Survey Paper on Scheduling in Hadoop". International Journal of Advanced Research in Computer Science and Software Engineering. 2014; 4.

[4] Dhruba Borthakur. "The Hadoop Distributed File System: Architecture and Design". The Apache Software Foundation. 2007.

[5] Yaxiong Zhao et al. "Dache: A Data AwareCaching for Big-Data Applications Using the MapReduce Framework". Tsinghua science and technology. 2014; 19(1), ISSN: I1007-0214I.

[6] Apache Pig. Available at http://pig.apache.org

[7] Apache Hive. Available at http://hive.apache.org

[8] Apache HBase. Available at http://hbase.apache.org 